\section{Temperature loggers are to be used to guarantee quality of insulin preparations}

Dear Sir,

Insulin preparations in powder form should be stored at $-20^{\circ} \mathrm{C}$ and in injection vials in a refrigerator between 2 and $8^{\circ} \mathrm{C}$. To maintain their biological activity injection vials should not be frozen during their storage [1]. Exposure of insulin vials for injection to sub-zero temperatures during refrigeration could freeze insulin and lower its potency. Subsequent injection of thawed insulin preparations could lower the applied dose inappropriately for the control of diabetes mellitus.

In Northern Australia continuous temperature control of 144 vials of hepatitis B vaccine unveiled that during their transportation to different immunisation centres, $47.5 \%$ of the vials had been exposed to temperatures of $-3^{\circ} \mathrm{C}$ or lower [2]; such handling could well apply to insulin vials. Thus, the unexpected exposure to low temperatures of insulin vials could occur quite frequently and add, by partial inactivation of insulin, to therapeutic instability in a climatic environment subject to un-

Corresponding author: S.C. Arya, Centre for Logistical Research and Innovation, M-122 (of part 2), Greater Kailash-II, New Delhi, 110048, India expected fluctuations. Breakdown of electricity supply to refrigeration facilities in deprived areas might add to the problems described [3]. It is for this reason that storage temperatures for insulin preparations would be best monitored using electronic loggers rather than thermometers. Loggers could be pre-set to record temperatures at half-hour intervals and any inadvertent temperature less than $0^{\circ} \mathrm{C}$ could be measured. Thereby insulin storage sites could be monitored and both the qualitative and quantitative deterioration in effective insulin potency determined.

Yours sincerely,

S.C. Arya

\section{References}

1. Physicians' Desk Reference (1997) 51st ed. Medical Economics Company, Montvale

2. Miller NC, Harris MF (1994) Are childhood immunization programmes in Australia at risk? Investigations of the cold chain in the Northern Territory. Bull World Health Organisation 72: 401-408

3. Kumar S (1998) India's heat wave and rains result in massive death toll. Lancet 351: 1869

\section{Muscle insulin resistance: a probable case of disuse of skeletal muscles. Lamarckism revisited}

\begin{abstract}
Dear Sir,
It was interesting to read two articles in the column 'For debates' on the thrifty genotype in the April 1998 issue of Diabetologia $[1,2]$. Assuming that acquired insulin resistance cannot be inherited, it is difficult to account for the familial clustering of insulin resistance as observed in Mexican American subjects with a parental history of diabetes [3] and in Pima Indians [4]. It would, therefore, be useful to know the basis of insulin resistance. Does it have a survival value? In vivo $[5,6]$ and in vitro $[7,8]$ studies found that the decrease in the whole body insulin-mediated glucose uptake in patients with Type II (non-insulin-dependent) diabetes mellitus is localized to a defect in the mode of insulin action at the level of skeletal muscle. Reaven [1] Wendorf and Goldfine and [9] have hypothesized that muscle insulin resistance probably represents the key feature that favoured the survival of primitive man characterized by the "thrifty genotype" proposed by Neel [10]. Muscle insulin resistance is seen not only in patients with Type II diabetes but also those with obesity, hypertension, cardiovascular diseases or syndrome X [11]. All these conditions are characterized by the common denominator of a change in lifestyle in caloric intake and physical activity coupled with cigarette smoking and alcohol abuse as part of the 'cocacolonization' or 'Westernization' process [12]. Conservation of the characteristics of primitive man is evident in rural populations with a low
\end{abstract}

Corresponding author: Dr. R.R. Bhonde, National Centre for Cell Science, Universitiy of Pune Campus, Ganeshkhind, Pune: 411 007, India incidence of Type II diabetes as opposed to urban populations exhibiting a high incidence of the disease in both developing and developed countries [13]. Indeed change in life style leading to a higher incidence of Type II diabetes and syndrome $\mathrm{X}$ disorder has been implicated by Barker [14] who linked the origin of the adult disease to the intrauterine environment, governed by the nutritional status of the mother during pregnancy, eventually leading to a low birth weight. In the majority of cases, low birth weight is attributed to under nourishment due to the lower socio-economic and cultural status of people in developing countries and the disadvantaged and minority groups in developed nations $[15,16]$. It can be argued that a child born with low birth weight may reverse the trend with an improved nutritional status coupled with a low physical activity thereby repeating the history of converting the primitive man to the modern man.

Comparison of the lifestyle of primitive or rural man with modern or urban man shows that the amount of physical activity is a decisive factor in use or disuse of skeletal muscles reminiscent of the classical Lamarckian theory on the role of use and disuse of organs in evolution [17]. In the context of the global epidemic of Type II diabetes [15], Lamarckian theory could be viewed with a changed perspective such that the disuse of muscles, due to decreased physical activity, has probably led to the present muscle insulin resistance. Insulin resistance is favourable to survival under conditions of poor nutrition. When fasting is replaced by feasting with concomitant change in the life style, however, disorders such as obesity and Type II diabetes $[1,11]$, emerge culminating in the evolution of muscle insulin resistance into a metabolic syndrome.

In fact, the desert gerbill Psammomys obesus ("Sand rat") a model of nutritionally induced insulin resistance and Type II diabetes $[18,19]$ clearly mimics the transition from primitive man to modern man. Genetic factors predispose the sand rat to Type II diabetes, whereas environmental, (i.e. lifestyle and nutritional) factors contribute to the expression of 